\title{
Distribuição espacial e estrutura populacional de Parkia platycephala Benth
}

\author{
Leovandes Soares da SILVA ${ }^{1 *}$, Cristiane Coelho de MOURA ${ }^{1}$, Diego dos Santos VIEIRA ${ }^{1}$, \\ Tatiano Ribeiro dos SANTOS ${ }^{2}$, Evandro Luiz Mendonça MACHADO ${ }^{1}$, Natália Viveiros SALOMÃO ${ }^{1}$ \\ ${ }^{1}$ Universidade Federal dos Vales do Jequitinhonha e Mucuri (UFVJM), Diamantina, MG, Brasil. \\ ${ }^{2}$ Profissional autônomo, Especialista em Educação para o Meio Ambiente, Bom Jesus, PI, Brasil. \\ *E-mail: leovandessoares@bol.com.br
}

Recebido em novembro/2018; Aceito em março/2019.

RESUMO: O objetivo conhecer o padrão espacial e a estrutura de duas populações de Parkia platycephala, em duas áreas no sul do Piauí. Realizou-se um censo de indivíduos que apresentaram pelo menos um fuste com diâmetro altura do peito (DAP) $\geq 5 \mathrm{~cm}$. Para tanto, as duas áreas amostradas foram divididas em faixas contendo 20 metros de largura. Dentro destas faixas, foram coletadas as coordenadas cartesianas o diâmetro dos indivíduos. Para o cálculo da distribuição espacial, foi aplicado a função K de Ripley univariada. Para a distribuição diamétrica foi utilizada intervalos de classes com amplitudes crescentes. Na área I foram amostrados 101 indivíduos e área basal de 7,744 $\mathrm{m}^{2} /$ ha, para a área II, 66 indivíduos e área basal de 4,654 $\mathrm{m}^{2} / \mathrm{ha}$. Os indivíduos da $P$. platycephala apresentou padrão de ocorrência agregado em ambas as áreas. Os indivíduos menores ocorrem próximos dos indivíduos maiores, formando população agregada. Em relação ao padrão espacial dos indivíduos por classe diamétrica predominou-se o aleatório, diferindo em intensidades, à medida que aumenta os diâmetros. As classes de diâmetro e altura revelam possíveis dificuldades de recrutamento, isso porque a maioria dos indivíduos estão acima de $10 \mathrm{~cm}$ de diâmetro e $6 \mathrm{~m}$ de altura respectivamente.

Palavras-chave: função K de Ripley; análise espacial; conservação.

\section{Spatial distribution and population structure of Parkia platycephala Benth}

\begin{abstract}
The objective was to know the spatial pattern and structure of two populations of Parkia platycephala, in two areas in the south of Piauí. A census was taken of individuals who had at least one bole with breast height diameter $(\mathrm{DBH}) \geq 5 \mathrm{~cm}$. To do so, the two areas sampled were divided into tracks containing 20 meters wide. Within these ranges, the Cartesian coordinates were the diameter of the individuals. To calculate the spatial distribution, the univariate Ripley K function was applied. For the diametrical distribution, class intervals with increasing amplitudes were used. In area I, 101 individuals and basal area of 7,744 $\mathrm{m} 2$ / ha were sampled, for area II, 66 individuals and basal area of 4,654 $\mathrm{m} 2$ / ha. The individuals of $P$. platycephala showed an aggregate occurrence pattern in both areas. Smaller individuals occur close to larger individuals, forming an aggregate population. In relation to the spatial pattern of the individuals by diametric class the random was predominant, differing in intensities, as the diameters increase. The diameter and height classes reveal possible recruitment difficulties, which is why most individuals are above $10 \mathrm{~cm}$ in diameter and $6 \mathrm{~m}$ in height, respectively.
\end{abstract}

Keywords: Ripley's K function; spatial analysis; conservation.

\section{INTRODUÇÃO}

A espécie Parkia platycephala Benth., conhecida popularmente como faveira, fava-de-bolota, visgueiro, favade-boi e sabiú, entre outros, pertence à família Leguminosae e subfamília Mimosoideae, ocorre principalmente nos domínios da Amazônia, Caatinga e Cerrado das regiões Norte e Nordeste do Brasil Forzza et al. (2010). É uma espécie de grande porte que atinge entre 8 e $18 \mathrm{~m}$ de altura. $\mathrm{O}$ tronco é curto e cilíndrico, apresenta casca rugosa e descamante. As folhas são duplamente compostas bipinadas medindo de 10-12 cm de comprimento. A inflorescência é disposta em capítulos globosos sobre pedúnculo pendentes Lorenzi (2002). Os frutos são classificados como legumes indeiscentes, geralmente são produzidos durante a estação seca, entre os meses de agosto a outubro (BULHÃO; FIGUEIREDO, 2002).

Segundo Alves et al. (2016), essa espécie possui potencial madeireiro, frequentemente é utilizada na fabricação de caixotaria, tabuados, pequenas construções, forros, confecção de brinquedos, além da lenha e carvão. Trata-se de uma espécie fornecedora de madeira comumente explorada e presente nos Planos de Manejo Florestal Sustentável (PMFS) da Amazônia (IBDF, 1987; NASCIMENTO et al., 2009).

Outro importante uso dessa espécie e à capacidade de tornar-se uma excelente fonte de suplementação alimentar para ruminantes Alves et al. (2007), uma vez que as vagens, quando maduras, constituem excelente fonte de alimentação, o que a torna de extrema importância para regiões onde há escassez de alimentos em períodos de seca. No estado do Piauí, a espécie $P$. platycephala possui ampla distribuição, agricultores utilizam as vagens maduras para suplementação de bovinos e caprinos durante o período da seca, período mais crítico, de menor disponibilidade de pastagem.

Apesar de ser uma espécie com elevada importância ecológica e potencial madeireiro, há uma imensa falta de conhecimento sobre sua estrutura e distribuição espacial. Uma vez que, conhecer o padrão espacial pode ser o primeiro passo 
para investigar as variáveis que define a configuração espacial e a distribuição natural das espécies (DALMASO et al., 2012).

Segundo Silva et al. (2008), a distribuição espacial mostra como os indivíduos se encontram organizados horizontalmente no ambiente. Uma vez que essa organização é resultado da combinação de fatores bióticos e abióticos, que atuam na dinâmica dos processos ecológicos das populações.

Por meio do padrão espacial, é possível avaliar como os processos ecológicos interferem no desenvolvimento de uma determinada espécie Pereira et al. (2006); Capretz et al. (2012); Bruzinga et al. (2013); Meira-Junior et al. (2017). Dentre estes processos, enfatiza-se a dispersão de sementes, a competição intra e interespecífica, heterogeneidade ambiental, herbivoria e doenças além da análise estrutural da comunidade em si (CONDIT et al. 2000; STOYAN; PENTTINEN, 2000; (DALMASO et al., 2012).

Portanto, conhecer o padrão espacial de espécies dentro da comunidade, é imprescindível para o planejamento, medidas de manejo sustentável e conservação de formações florestais Arruda; Daniel (2007). Auxilia também nas estratégias de manejo, importantes na definição de metodologias de amostragem e em estudos de Ecologia (ARAÚJO et al., 2014).

Diante dessas informações, compreender a distribuição espacial e estrutura dos fragmentos florestais é uma das formas importantes para estabelecer estratégias de conservação. Desta forma, objetivou-se neste estudo a análise do padrão espacial e estrutural da espécie $P$. platycephala Benth., em duas áreas no Sul do Piauí.

\section{MATERIAL E MÉTODOS}

\section{1. Áreas do estudo}

O estudo foi realizado em duas áreas de propriedades particular, inseridas na bacia do Rio Gurguéia, na zona rural do município de Bom Jesus, no Sul do Piauí. As áreas do estudo se encontram em ecótono Cerrado/Caatinga. Área I (AI) localiza-se nas coordenadas $9^{\circ} 13^{\prime} 9.74^{\prime \prime} \mathrm{S}$ e $44^{\circ} 26^{\prime} 22.10^{\prime \prime} \mathrm{O}$, altitude $270 \mathrm{~m}$, e possui aproximadamente 10 hectares. Área II (AII) se encontra nas coordenadas $9^{\circ} 13^{\prime} 21.95^{\prime \prime} \mathrm{S}$ e $44^{\circ} 26^{\prime} 20.93^{\prime \prime} \mathrm{O}$, altitude $268 \mathrm{~m}$ e possui aproximadamente 9 hectares.

Nas duas áreas o relevo é plano com textura arenosa, os solos foram classificados de forma geral como Neossolos Quartzarênicos (SANTOS et al., 2011).

O clima da região de acordo com Köppen é classificado como Aw, temperatura média $26,7^{\circ} \mathrm{C}$ e pluviosidade média anual de $1002 \mathrm{~mm}$ Alvares et al. (2013), apresenta duas estações bem definidas sendo, a estação seca que compreende os meses de Maio a Outubro, temperatura média $27^{\circ} \mathrm{C}$ e precipitação média $22 \mathrm{~mm}$. Na estação chuvosa que ocorre de Novembro a Abril, a temperatura média é de $26,4^{\circ} \mathrm{C}$ e precipitação média $148 \mathrm{~mm}$, disponível em: (<https://pt.climate-data.org $>$ acessado em fev 2018).

As duas áreas presentam histórico de perturbações antrópicas (corte seletivo de algumas espécies), e trânsito de gado. Ambas circundadas por áreas antropizadas, compostas por lavouras e pastagens abandonadas.

\subsection{Coleta de dados}

Os dados foram coletados por meio de um inventário de prospecção (censo), com mapeamento em coordenadas cartesianas para todos os indivíduos que apresentaram pelo menos um fuste com diâmetro a 1,30 m do solo (DAP) igual ou superior a $5 \mathrm{~cm}$. Para este procedimento, cada área foi dividida em faixas contendo 20 metros de largura. Dentro das faixas, realizou-se o caminhamento em 'zig-zag'. Os indivíduos a partir do diâmetro de inclusão foram plaqueteados, para garantir que não fossem mensurados mais de uma vez, e para o monitoramento destes ao longo do tempo.

\subsection{Classes diamétricas}

A distribuição das classes de diâmetro foi realizada por meio de intervalos com amplitudes crescentes, como adotado em estudos de Appolinário et al. (2005). As classes diamétricas foram divididas da seguinte forma: classe 1 - de 5 a $10,0 \mathrm{~cm}$ de DAP; classe 2 - de 10,1 a 20,0cm de DAP; classe 3 - de 20,1 a $40,0 \mathrm{~cm}$ de DAP e classe 4 acima de $40 \mathrm{~cm}$ de DAP. Isso, para compensar o forte decréscimo da densidade nas classes de tamanhos maiores, típico da distribuição em exponencial negativo.

Esse trabalho foi realizado no mês de setembro, época que segundo Kuhlmann et al. (2018), a espécie P. platycephala se encontra com frutos e sementes maduras. Assim, foi observado em campo a presença (indivíduos com frutos- $\mathrm{CF}$ ), ou ausência - SF) de frutos.

Para as classes de altura, foi empregado intervalos fixos de $3 \mathrm{~m}$ para todos os indivíduos amostrados nas áreas do estudo.

\subsection{Distribuição espacial}

A análise da distribuição espacial foi feita para toda a população, dividida nas seguintes classes: classe 1 - até 11,00 cm de DAP; classe 2 - de 11,01 a 22,00 cm de DAP; classe 3 de 22,01 a 30,00 $\mathrm{cm}$ de DAP e classe 4 - acima de 30,00 $\mathrm{cm}$ de DAP (SANTANA; NAVES, 2003; BRUZINGA et al., 2013).

Determinou-se o padrão espacial empregando a função $\mathrm{K}$, proposta por Ripley (1976), que considera a variância de todas as distâncias entre todos os eventos.

A função K(s) de Ripley foi obtida por meio de um círculo com raio $s$ de $5 \mathrm{~m}$ centrado em cada árvore, em que o número de vizinhos presentes na área desse círculo foi contado (Figura $1)$.

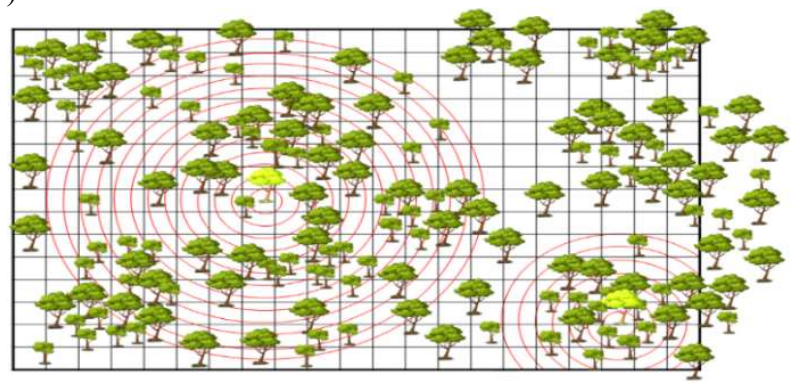

Figura 1. Ilustração do procedimento de obtenção da função K(s) de Ripley.

Figure 1. Illustration of the procedure of obtaining the function K (s) of Ripley.

Variando o raio $s$ a uma distância máxima de 195 e 180m para a área de ocorrência da espécie (I e II) respectivamente, detectou-se o padrão espacial da espécie em diferentes escalas de distância (VIEIRA et al., 2017).

Casos particulares ocorreram quando as árvores estavam próximo às bordas dos fragmentos, uma vez que, por ser a função $\mathrm{K}(\mathrm{s})$ acumulativa e computa todas as distâncias entre todos os eventos, as árvores próximas à borda de raio $s$ maior que o limite do mapa não poderiam ser interpretadas como se não houvesse vizinhos Capretz et al. (2012). Os vizinhos 
existiam, mas por estarem fora dos limites da área não foram computados. Consequentemente, o número de árvores vizinhas as árvores próximas aos limites do mapa seriam mais baixo do que para as demais, ocasionando um viés no cálculo do estimador da função K(s) de Ripley. À vista disso, utilizouse o estimador da função $\mathrm{K}$ com correção isotrópica de bordadura (RIPLEY, 1977).

$K(\mathrm{~s})=\frac{1}{\hat{\lambda}_{\mathrm{n}}} \sum_{\mathrm{i}=1}^{\mathrm{n}} \sum_{\mathrm{i}=1}^{\mathrm{n}} \frac{1}{\mathrm{~W}_{\mathrm{I}}}\left(\mathrm{x}_{\mathrm{i}}, \mathrm{x}_{\mathrm{j}}\right) \mathrm{I}\left(\left\|\mathrm{x}_{\mathrm{i}}-\mathrm{x}_{\mathrm{j}}\right\|<\mathrm{s}\right)$, para $\mathrm{i} \neq \mathrm{j}$ e $\mathrm{S}>0$

em que: $n$ é o número de árvores na região de estudo; $\mathrm{X}_{i}$ e $\mathrm{X}_{j}$ são as coordenadas dos pontos do mapa; $\left\|\mathrm{X}_{\mathrm{i}}-\mathrm{X}_{\mathrm{j}}\right\|$ é a distância euclidiana entre a localização $\mathrm{X}_{i}$ e $\mathrm{X}_{j} ; s$ é um vetor arbitrário de distância; $\mathrm{W}_{\mathrm{I}}$ $\mathrm{I}\left(\mathrm{X}_{i}, \mathrm{X}_{j}\right)$ é a função de correção para efeito de borda, que representa a proporção da circunferência com centro em $X_{i}$ e com raio $\left\|X_{i}-X_{j}\right\|$ que está fora da região de estudo; $\hat{\lambda}=\mathrm{n} /|\mathrm{A}|$ é o número de árvores dividido pela área da região de estudos, sendo um estimador não viciado da intensidade do processo; e I(U) é uma função indicadora que assume valor 1 sempre que a condição $U$ for verdadeira e zero quando for falsa.

Em seguida, objetivando analisar os dados graficamente foram construídos envelopes de confiança por meio de 1.000 simulações Monte Carlo, realizadas com o modelo de completa aleatoriedade espacial (CAE), testou-se hipóteses a um nível de confiança de 95\%. Posteriormente, foi calculada a função K(s) para os resultados das simulações, armazenandose os valores mínimos e máximos da estimativa de $\mathrm{K}(\mathrm{s})$, utilizados para gerar intervalos de confiança a $99 \%$ de probabilidade. Para facilitar a análise, os valores da função $\mathrm{K}$ (s) foram transformados para $\mathrm{L}(\mathrm{s})$, de acordo com a formulação apresentada a seguir, e distribuídos em um gráfico, em que os eixos das abscissas e ordenadas representam, respectivamente, as distâncias $s$ acumuladas e os valores transformados da função K (RIPLEY, 1979).

$L(s)=\sqrt{\frac{K(s)}{\pi}}-s$

Como resultado, foram obtidos envelopes de confiança identificado por duas linhas limites pontilhadas, uma positiva e outra negativa. Se os valores observados de L(s) estiverem dentro dos envelopes construídos, o padrão espacial é aleatório, caso contrário rejeita-se a hipótese nula e assume-se que o padrão espacial dos indivíduos é agregado, quando passar do limite superior do envelope, e regular, ao exceder o limite inferior (RIPLEY, 1979; MACHADO et al., 2012).

Os cálculos foram realizados com o auxílio do software $\mathrm{R}$ versão 3.3.2, sendo as análises da função $\mathrm{K}$ de Ripley por meio do pacote splancs (ROWLINGSON; DIGGLE, 2017).

\section{RESULTADOS}

Por meio do censo, na (AI) foram mensurados um total de 101 indivíduos e área basal de 7,744 m²/ha, na (AII), 66 indivíduos e área basal de 4,654 $\mathrm{m}^{2} / \mathrm{ha}$. Analisando a distribuição diamétrica, a maioria dos indivíduos estão em maior frequência distribuídos na segunda e terceira classe, respectivamente. '̀ medida que aumentou o diâmetro verificou-se um acréscimo nas frequências dos indivíduos (Figura 2).

$\mathrm{Na}(\mathrm{AI})$, o (DAP) variou de 5 a $113 \mathrm{~cm}$, os indivíduos apresentaram-se concentrados na terceira classe $(39,6 \%)$, seguidos da primeira classe $(22,77 \%)$ (Figura 2, AI). Já na
(AII) o (DAP) variou de 8,1 a $61,1 \mathrm{~cm}$, a maioria dos indivíduos $(50 \%)$ estão concentrados na terceira classe, seguidos da segunda classe (28,78\%), (Figura 2, AII).
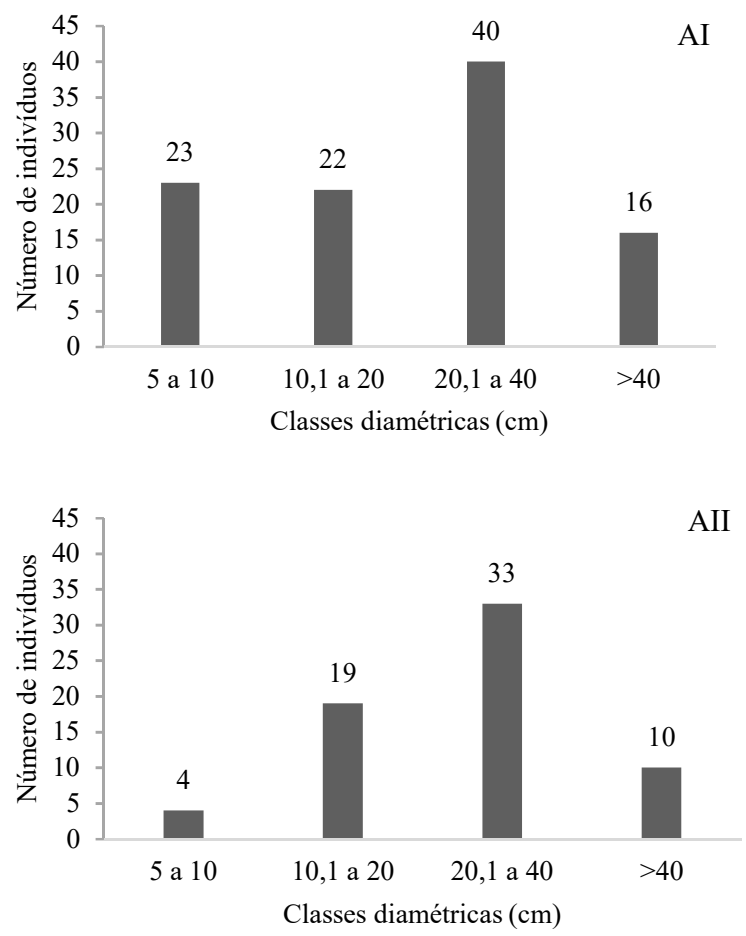

Figura 2. Distribuição diamétrica dos indivíduos de Parkia platycephala em duas áreas no sul do Piauí.

Figure 2. Diametric distribution of the individuals of Parkia platycephala in two areas in the south of Piauí.
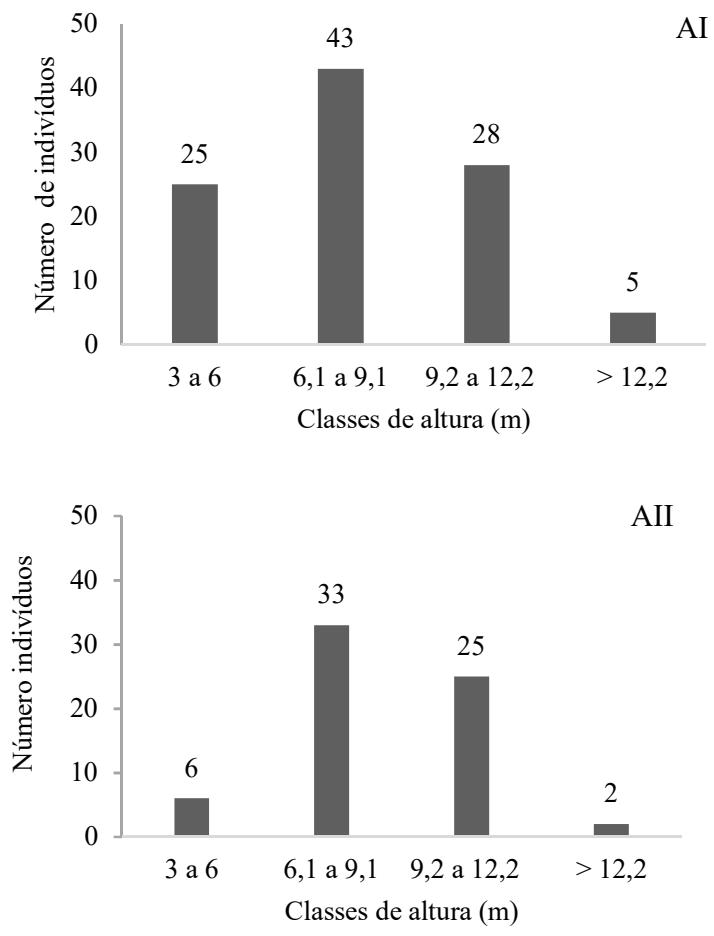

Figura 3. Número de indivíduos de Parkia platycephala por classes de altura, amostrados em duas áreas de ecótono Cerrado/Caatinga no município de Bom Jesus, Piauí.

Figure 3. Number of individuals of Parkia platycephala by height classes, sampled in two Cerrado/Caatinga ecotone areas in the municipality of Bom Jesus, Piauí. 
$\mathrm{Na}$ distribuição por classes de altura na (AI), a primeira classe altura dos indivíduos variou de 3,0 a $6,0 \mathrm{~m}$, e assim sucessivamente. A maioria dos indivíduos se encontram na segunda e terceira classe, juntas representam 70,2\% dos indivíduos, a média de altura encontrada foi de $7,9 \mathrm{~m}$.

$\mathrm{Na}$ (AII), a primeira classe altura variou de 3,2 a $6,2 \mathrm{~m} \mathrm{e}$ assim, sucessivamente. A maioria dos indivíduos também se
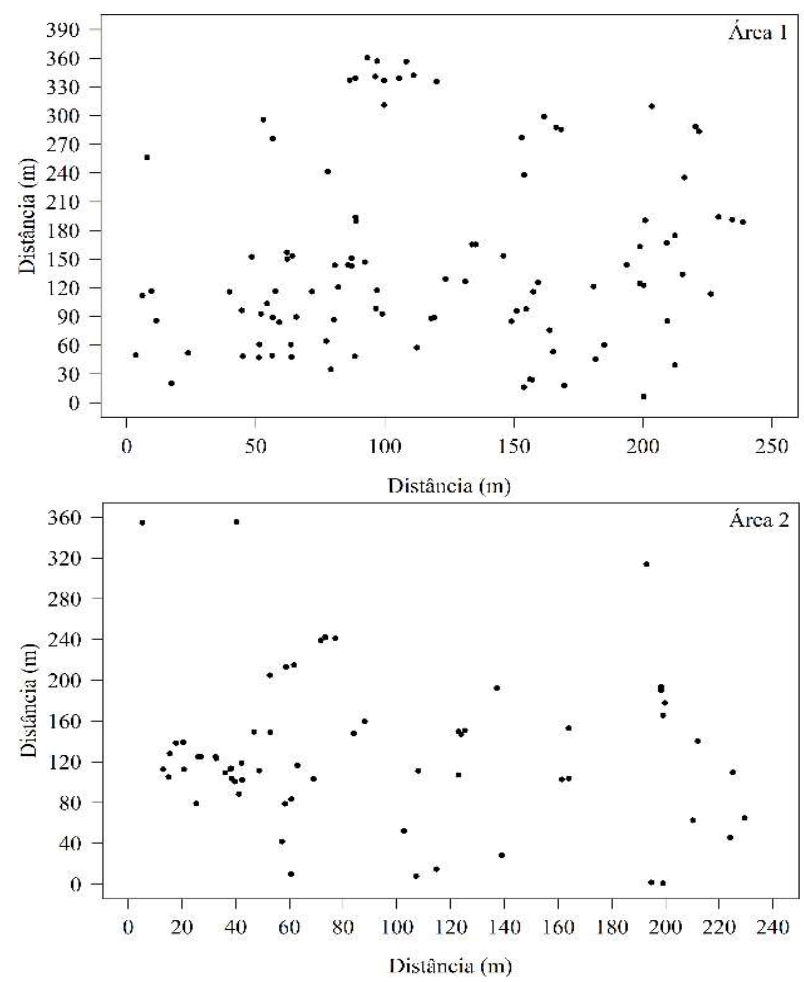

encontram na segunda e terceira classe, juntas representam $87,8 \%$ dos indivíduos, altura média foi de $8,4 \mathrm{~m}$ (Figura 3). $\mathrm{O}$ mapa com a orientação de cada indivíduo de P. platycephala e seus respectivos gráficos da função K de Ripley, para cada área encontra representado na Figura 4.
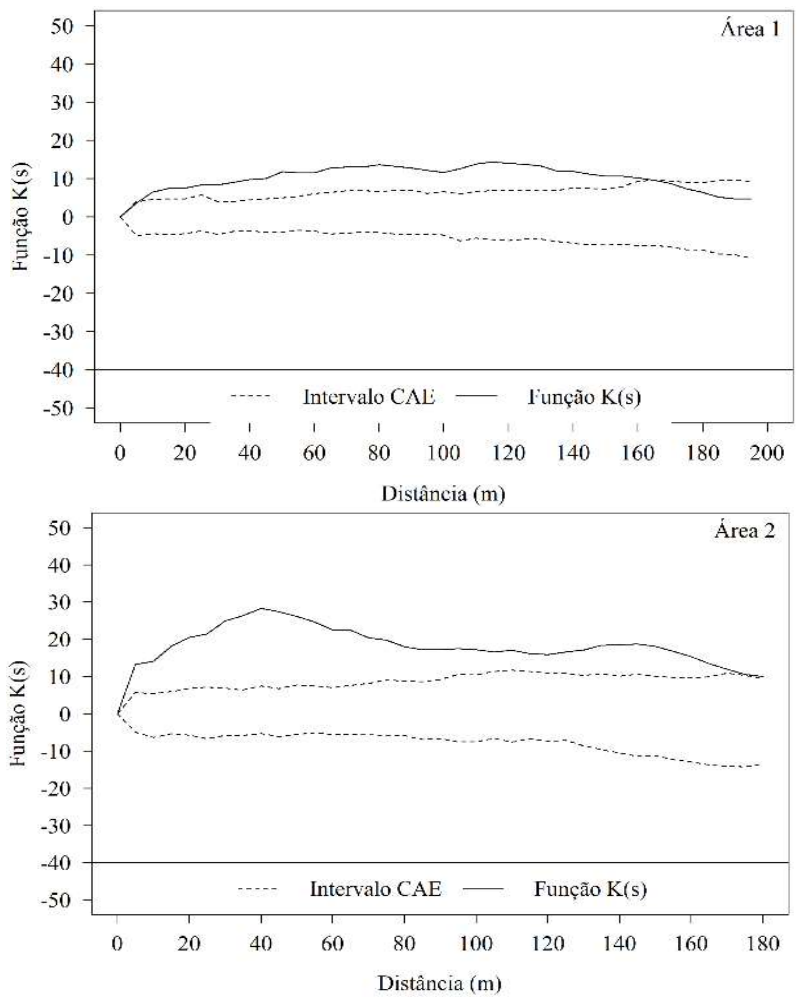

Figura 4. Mapa da distribuição espacial da população total de Parkia platycephala. As linhas tracejadas indicam os envelopes de confiança pelas simulações do Método Monte Carlo a 99\%, para a Completa Aleatoriedade Espacial (CAE). As linhas contínuas demonstram o ajuste pela Função K de Ripley transformado (L(s)) AI - Área I e AII - Área II.

Figure 4. Map of the spatial distribution of the total population of Parkia platycephala. Dashed lines indicate the reliable envelopes by the 99\% Monte Carlo Method simulations for Complete Spatial Randomness (CAE). The continuous lines demonstrate the adjustment by the transformed Ripley K Function (L (s)) AI - Area I and AII - Area II.

Nas duas áreas, considerando toda a população, rejeitou-se a hipótese de (CAE). A população de $P$. platycephala apresentou padrão de distribuição espacial agregado até a escala de $170 \mathrm{~m}$ na (AI), a partir de $170 \mathrm{~m}$ a distribuição foi aleatória, o padrão espacial difere em intensidades de agregações, para as diferentes distâncias. Enquanto que na (AII), o padrão espacial foi agregado (Figura 4).

Por classes diamétricas, em sua totalidade, a população de $P$. platycephala apresentou padrão aleatório, em todas as classes diamétricas, diferindo em intensidades de agregações para a área AI (Figura 5). Em relação à AII, a distribuição dos indivíduos por classes diamétricas variaram entre agregado e aleatório (Figura 5, AII).

$\mathrm{Na}$ (AII), a classe de até $11 \mathrm{~cm}$ a distribuição foi aleatória (Figura 5), isso pode ser explicado por se tratar de uma classe com poucos indivíduos. Na segunda classe (diâmetros entre 11,1 a $22 \mathrm{~cm}$ ) a distribuição foi agregado até escala de $70 \mathrm{~m}$, a partir desse ponto a distribuição seguiu de forma aleatória variando em intensidades (Figura 5). Na terceira classe (22,1 a $30 \mathrm{~cm}$ ), os indivíduos se comportaram de forma aleatória até $40 \mathrm{~m}$, agregado entre 40 e $85 \mathrm{~m}$, a partir desse ponto a distribuição voltou ao padrão aleatório. Enquanto na classe acima de $30 \mathrm{~cm}$ o padrão foi totalmente aleatório, quanto maior o diâmetro, mais aleatório se encontram os indivíduos. Conforme a Tabela 1 , na (AI) 25,74\% dos indivíduos se encontravam com frutos e $74,25 \%$ sem frutos. Já na (AII), $37,87 \%$ com frutos e $62,12 \%$ sem frutos.

A Tabela 1, mostra que, em todas as classes diamétricas os indivíduos produzem frutos, exceto na primeira classe da (AII), pode ser decorrente da baixa quantidade de indivíduos nessa referida classe. Abaixo de $40 \mathrm{~cm}$ de diâmetro, a quantidade de indivíduos sem fruto é superior aos com frutos, enquanto acima de $40 \mathrm{~cm}$ de diâmetro, a maioria produz frutos.

Tabela 1: Número de indivíduos de Parkia platycephala amostrados em duas áreas de ecótono Cerrado/Caatinga, em Bom Jesus, Piauí.

Table 1: Number of Parkia platycephala individuals sampled in two Cerrado / Caatinga ecotone areas in Bom Jesus, Piauí.

\begin{tabular}{|c|c|c|c|c|}
\hline \multirow{2}{*}{ Classes diamétricas } & \multicolumn{2}{|c|}{$\mathrm{AI}$} & \multicolumn{2}{|c|}{ AII } \\
\hline & $\mathrm{Cf}$ & Sf & $\mathrm{Cf}$ & $\mathrm{Sf}$ \\
\hline 5 a 10 & 4 & 19 & 0 & 4 \\
\hline 10,1 a 20 & 3 & 19 & 1 & 18 \\
\hline 20,1 a 40 & 7 & 33 & 15 & 18 \\
\hline$>40$ & 12 & 4 & 9 & 1 \\
\hline Total & \multicolumn{2}{|c|}{101} & \multicolumn{2}{|c|}{66} \\
\hline
\end{tabular}

em que: $\mathrm{Cf}=$ com fruto e $\mathrm{Sf}=$ sem fruto; $\mathrm{In}$ what: $\mathrm{Cf}=$ fruit and $\mathrm{Sf}=$ fruit. 

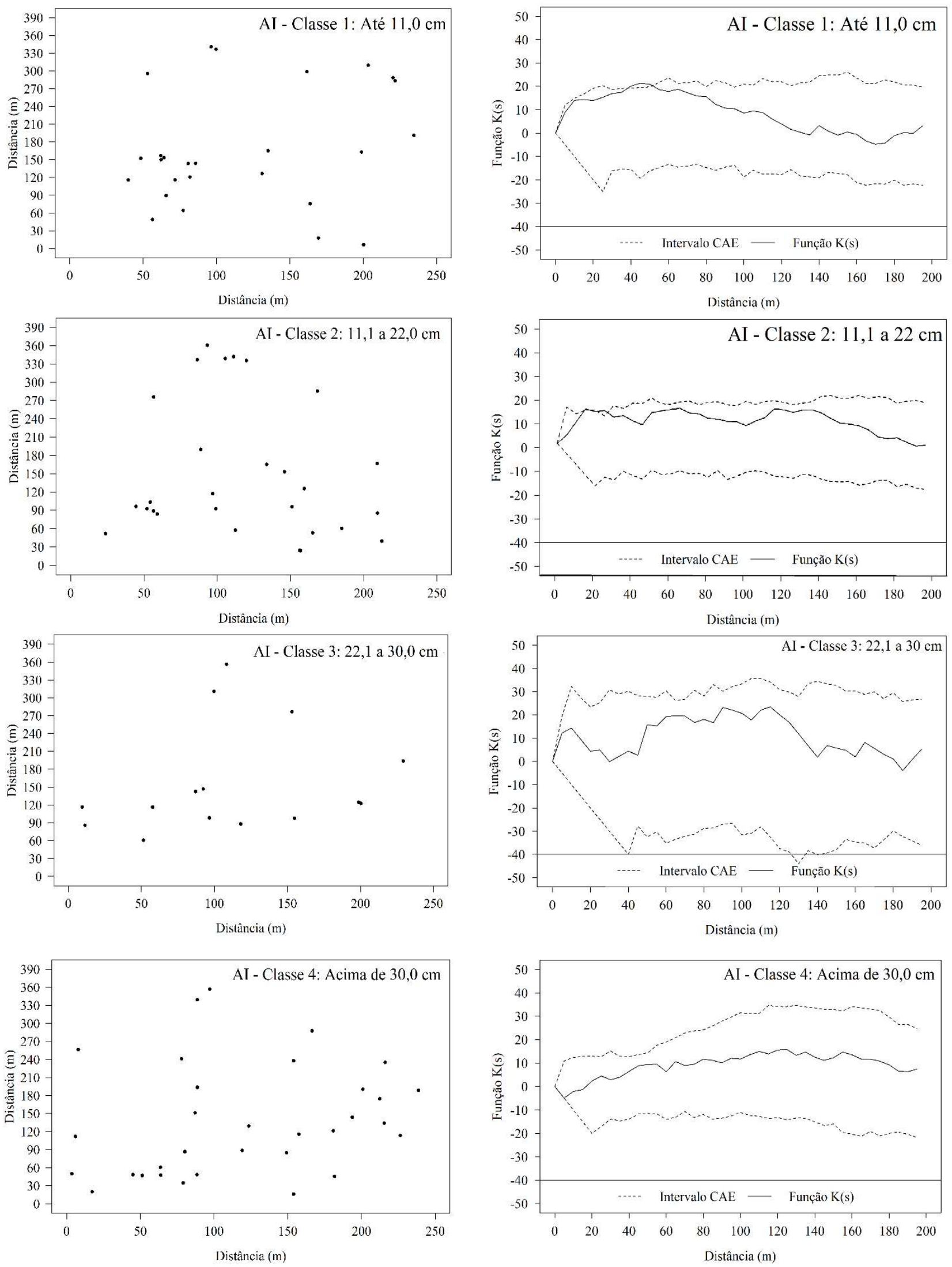

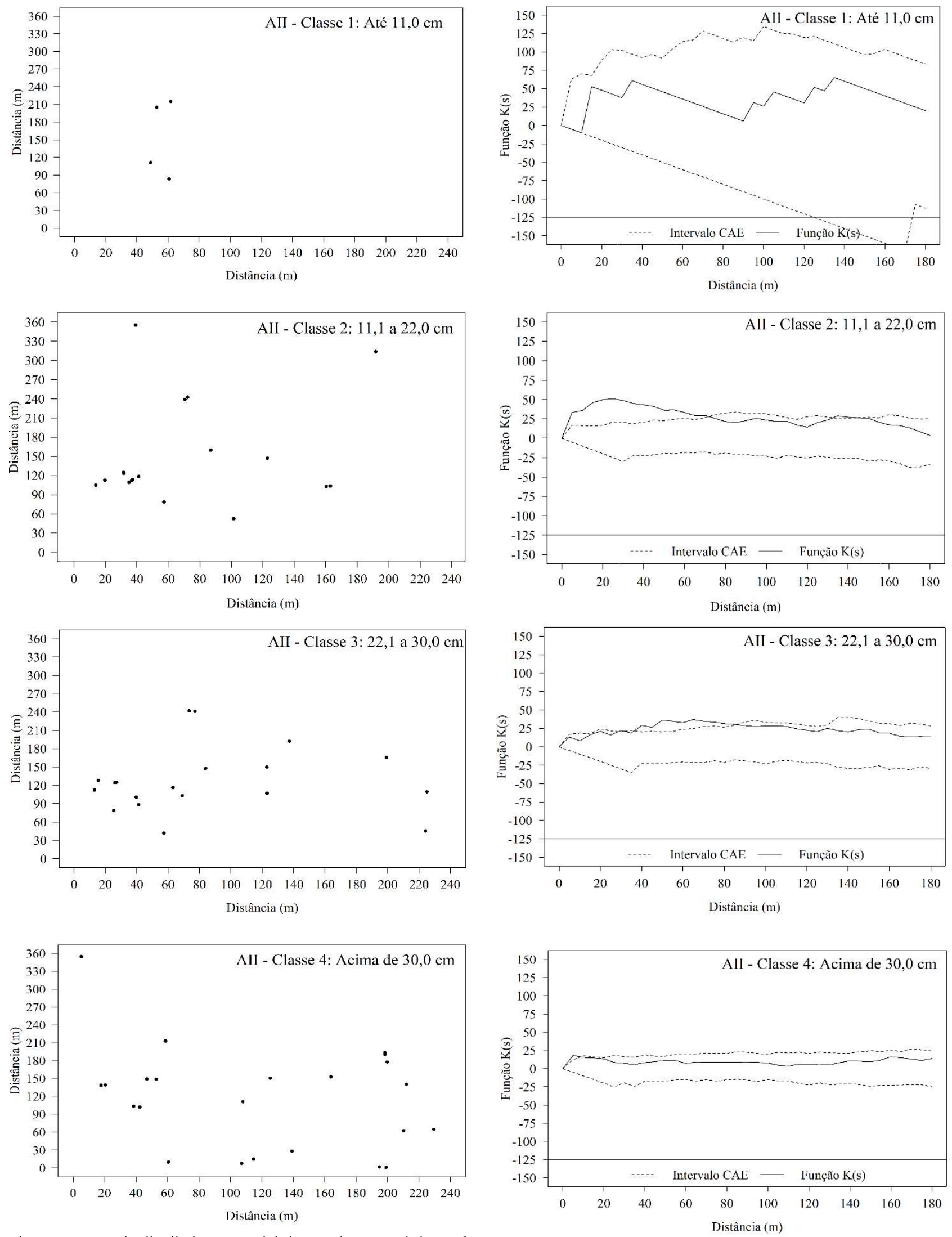

Figura 5. Mapa da distribuição espacial da população total de Parkia

platycephala. Função Ripley K (L estimado) em diferentes escalas para os indivíduos por classes diamétrica. As linhas tracejadas indicam os envelopes de confiança pelas simulações do Método Monte Carlo a 99 \%, para a Completa Aleatoriedade Espacial (CAE). As linhas contínuas demonstram o ajuste pela Função K de Ripley transformado (L(s)) AI - Área I e AII - Área II.

Figure 5. Map of the spatial distribution of the total population of Parkia platycephala. Function Ripley K (L estimated) at different scales for individuals by diameter classes. The dashed lines indicate the reliable envelopes by the $99 \%$ Monte Carlo Method simulations for Complete Spatial Randomness (CAE). The continuous lines demonstrate the adjustment by the transformed Ripley K Function (L (s)) AI - Area I and AII - Area II. 


\section{DISCUSSÃO}

As duas áreas apresentam irregularidade entre as classes diamétricas, provavelmente seja resultado de processo natural de sucessão, tendo em vista que a maioria dos indivíduos possuem diâmetros superiores a $20 \mathrm{~cm}$.

Foi observado, em campo, pouquíssimos indivíduos com diâmetro abaixo de $5 \mathrm{~cm}$, possivelmente seja por que essa espécie apresenta dormência nas sementes, dificultando assim sua propagação Nascimento et al. (2009). A dormência impede a germinação durante períodos adversos, com isso, aumenta a longevidade das sementes, previne as sementes na eventualidade ocorrência de chuvas esporádicas comuns durante a estação seca Santos et al. (2015). Ou porque ruminantes se alimentam das vagens, já que é comum a presença desses animais nessas áreas há bastante tempo, dificultando assim o nascimento e crescimento de novos indivíduos dessa espécie. Uma vez que, a presença de animais de grande porte pode favorecer e mortalidade de plântulas. Assim, futuramente, as duas áreas poderão apresentar problemas de estabelecimento de novos indivíduos, isso, poderá implicar no insucesso reprodutivo das populações futura (BARBOSA et al., 2017).

Uma vez que com exceção da primeira classe diamétrica (AII), todas as outras classes possuem indivíduos reprodutivos de sementes, importantes para manutenção e continuidade da população Tabela 1. Conforme Dorneles; Negrelle (2000) os indivíduos das maiores classes são reprodutivamente maduros e potencialmente responsáveis pela continuidade do processo dinâmico através da produção de sementes, enquanto os indivíduos das menores classes são os únicos responsáveis que garantem a manutenção da população, embora são estes que sofrem as maiores taxas de mortalidade. Em geral, nos ambientes naturais esse efeito é esperado, devido à competição entre plântulas ou pisoteio de animais de grande porte

A Tabela 1 mostra que, indivíduos em classes de tamanho intermediárias podem ser reprodutivos e contribui para a continuidade da população. Nas duas áreas, a maioria dos indivíduos possuem altura superior a $6 \mathrm{~m}$, uma vez que a caracterização de altura permite inferir que as populações podem ser consideradas auto-perpetuantes, pois, os indivíduos se apresentam com potenciais de desenvolvimento, das menores para as maiores classes de altura, respectivamente (ODUM, 1988).

Considerando a população total, o padrão de distribuição encontrado foi o agregado. Essa forma de distribuição pode ser advinda da limitação de dispersão, competição intra ou interespecífica, heterogeneidade do habitat, distribuição do recurso em manchas ou padrão de distribuição de sementes (CAPRETZ et al., 2012; SONG et al., 2017).

A síndrome de dispersão pode influenciar na distribuição espacial dos indivíduos de determinada espécie, que pode se comportar de forma agregada, aleatória ou uniforme Negrini et al. (2012). P. platycephala possui dispersão barocórica, isso explicaria o padrão de distribuição espacial agregado, ou seja, a germinação das sementes próximas às árvores-matrizes. Segundo Araújo et al. (2014), as variações nos padrões de distribuição podem estar relacionadas ao porte arbóreo dos indivíduos, sendo que indivíduos mais jovens tendem a ocorrer mais próximos uns dos outros. Por estarem em processo de recrutamento Capretz et al. (2012). Outros fatores a serem considerados na distribuição dos indivíduos são as condições edáficas e topográficas local (QIN et al., 2017).
A medida que esses indivíduos jovens competem entre si ou com indivíduos de outras espécies, pode ocorrer maior porcentagem de mortalidade nessa fase, isso, depende da característica de cada espécie, ou seja, para atingir a fase adulta, os indivíduos são selecionados naturalmente.

Por classes damétrica nas duas áreas a distribuição dos indivíduos tenderam a um padrão de distribuição aleatório (Figura 5), exceto a segunda classe (árvores entre 11,1 a 22cm) na (AII), em que a distribuição foi agregada até escala de $70 \mathrm{~m}$. Esse padrão indica independência espacial entre os indivíduos (ARAÚJO et al., 2014).

A maioria dos indivíduos estão nas classes de maiores diâmetros, conforme os resultados apresentados nas figuras, quanto maior o diâmetro, mais aleatório os indivíduos ficam mais distantes uns dos outros. Segundo Townsend et al. (2010), isso ocorre em ambientes com recursos limitados devido à saturação de sítios, ou pela forma de dispersão da espécie. A junção de múltiplos pequenos fatores influenciam no padrão de distribuição espacial, isso pode resultar em um padrão aleatório Odum, (2010). Muitos indivíduos não alcançam diâmetros maiores, seja pela mortalidade causada pela competição na fase jovem, ou pelo próprio ciclo de vida da espécie, à medida que crescem ficam distribuídos aleatoriamente, como observados na distribuição de outras espécies (BRUZINGA et al., 2013; ARAÚJO et al., 2014).

$\mathrm{O}$ padrão de distribuição foi variado na (AI), todas as classes apresentaram padrão aleatório bem definido nas maiores escalas, já na (AII) variou de agregado a aleatório, dependendo da escala de distância, o padrão aleatório ficou bem definido para os indivíduos de maiores diâmetros. Já Bruzinga et al. (2013) avaliaram a distribuição espacial de Caryocar brasiliensis encontraram padrão completamente agregado para indivíduos com DAP acima $30 \mathrm{~cm}$. Segundo Bruzinga et al. (2013), alguns fatores podem influenciar no padrão de distribuição espacial de uma espécie, como, por exemplo, os aspectos reprodutivos. O padrão espacial se explica através das características de cada espécie, como por exemplo, a fenologia, síndromes de dispersão e polinização, estratégias de ocupação de habitats disponíveis e potencial competitivo (CAPRETZ et al., 2012).

A espécie $P$. platycephala produz muitos frutos e sementes. Possui rápido crescimento Alves et al. (2016). Apresenta excelente potencial madeireiro e fonte de alimentação para ruminantes (ALVES et al., 2007).A dormência e a presença de ruminantes, animais de grande porte, pode estar influenciando a propagação dessa espécie nessas áreas. Pretende-se avaliar o desenvolvimento desses indivíduos e dos possíveis ingressantes em intervalos de tempo determinado, para ver se esse comportamento observado se manterá com o tempo.

\section{CONCLUSÕES}

Nas duas áreas as populações de $P$. platycephala se distribuíram de forma agregada. Já por classes diamétricas a distribuição variou de agregada a aleatória.

Em todas as classes de diâmetro a distribuição espacial aleatória foi bem definida (AI). O padrão de distribuição foi aleatório para indivíduos até $11 \mathrm{~cm}$ e acima de $30 \mathrm{~cm}$ de diâmetro, nas outras classes variou de agregado e aleatório em diferentes escalas de distâncias (AII).

As classes de diâmetro e altura revelam possíveis dificuldades no recrutamento de novos indivíduos, isso porque 
a maioria dos indivíduos estão acima de $10 \mathrm{~cm}$ de diâmetro e $6 \mathrm{~m}$ de altura respectivamente (AI e AII).

\section{REFERÊNCIAS}

ALVARES, C. A.; STAPE, J. L.; SENTELHAS, P. C.; GONÇALVES, J. L. de M.; SPAROVEK, G. Köppen's climate classification map for Brazil. Meteorologische Zeitschrift, Berlin, v. 22, n. 6, p. 711-728, 2013 https://dx.doi.org/10.1127/0941-2948/2013/0507

ALVES, A. A.; SALES, R. O.; NEIVA, J. N.; MEDEIROS, A. N.; BRAGA, A. P.; AZEVEDO, A. R. Degradabilidade ruminal in situ de vagens de faveira (Parkia platycephala Benth.) em diferentes tamanhos de partículas. Revista Brasileira de Medicina Veterinária e Zootecnia, Belo Horizonte, v. 59, n. 4, p. 1045-1051, 2007. DOI: http://dx.doi.org/10.1590/S0102-09352007000400034

ALVES, L. R.; OLIVEIRA, R. J.; COIMBRA, R. R.; FERREIRA, W. M. Crescimento inicial de Parkia platycephala (Benth.) e Enterolobium timbouva (Mart.) sob condições de campo numa área de Cerrado. Revista Ceres, Viçosa, v. 63, n. 2, p. 154-164, 2016. https://dx.doi.org/10.1590/0034-737X201663020006

APPOLINÁRIO, V.; OLIVEIRA-FILHO, A. T.; GUILHERME, F. A. G. Tree population and community dynamics in a Brazilian tropical semideciduous forest. Revista Brasileira de Botânica, São Paulo, v. 28, n. 2, p. 347-360, 2005. DOI: http://dx.doi.org/10.1590/S010084042005000200014

ARAÚJO, E. J. G.; DAVID, H. C.; NETTO, S. P.; MORAIS, V. A.; SCOLFORO, J. R. S. Padrão espacial de espécies arbóreas em fragmento de floresta estacional semidecidual. Revista de Ciências Agrárias, Belém, v. 57, n. 2, p. 166-171, 2014. DOI: http://dx.doi.org/10.4322/rca.2014.010

ARRUDA, L.; DANIEL, O. Florística e diversidade em um fragmento de floresta estacional semidecidual aluvial em Dourados-MS. Floresta, Curitiba, v. 37, n. 2, p. 189-199, 2007. DOI: http://dx.doi.org/10.5380/rf.v37i2

BARBOSA, A. S.; ANDRADE, A. P.; PEREIRA JÚNIOR, L. R.; BRUNO, R. L. A.; MEDEIROS, R. L. S.; BARBOSA NETO, M. A. Estrutura populacional e espacial de Cereus jamacaru DC. Em duas áreas de caatinga do agreste da Paraíba, Brasil. Ciência Florestal, Santa Maria, v. 27, n. 1, p. 315-324, 2017. http://dx.doi.org/10.5902/1980509826469

BRUZINGA, J. S.; OLIVEIRA, M. L. R.; MACHADO, E. L. M.; LEITE, H. G.; PEREIRA, I. M.; NOGUEIRA, G. S. Distribuição espacial de indivíduos adultos de pequi. Scientia Forestalis, Piracicaba, v. 41, n. 98, p. 249-256, 2013.

BULHÃO, C. F.; PAULO S. FIGUEIREDO, P. S. Fenologia de leguminosas arbóreas em uma área de cerrado marginal no nordeste do Maranhão. Revista Brasileira de Botânica, São Paulo, v. 25, n. 3, p. 361-369, 2002. DOI: http://dx.doi.org/10.1590/S0100-84042002000300012

CAPRETZ, R. L.; BATISTA, J. L. F.; SOTOMAYOR, J. F. M.; CUNHA, C. R.; NICOLETTI, M. F.; RODRIGUES, R. R. Padrão espacial de quatro formações florestais do estado de São Paulo, através da função $\mathrm{K}$ de Ripley. Ciência Florestal, Santa Maria, v. 22, n. 3, p. 551-565, 2012. DOI: http://dx.doi.org/10.5902/198050986622

CONDIT, R.; ASHTON, P. S.; BAKER, P.; BUNYAVEJCHEWIN, S.; GUNATILLEKE, S.;
GUNATILLEKE, N.; HUBBELL, S. P.; FOSTER, R. B.; ITOH, A.; LAFRANKIE, J. V.; LEE, H. S.; LOSOS, E.; MANOKARAN, N.; SUKUMAR, R.; YAMAKURA, T. Spatial Patterns in the Distribution of Tropical Tree Species. Science, v. 288, n. 5470, p. 1414-1418, 2000. DOI: https://dx.doi.org/10.1126/science.288.5470.1414

DALMASO, C. A.; INOUE, M. T.; OLIVEIRA, P. C. F. O.; MARCELINO, V. R.; POTTKER, G. S. Análise dos padrões espaciais de Ocotea odorifera (Vell.) Rohwer na Floresta Nacional de Irati (PR). Revista Ambiência, Guarapuava, v. 8, n. 4, p. 559-570, 2012. DOI: http://dx.doi.org/10.5777/ambiencia.2012.04.10

DORNELES, L. P. P.; NEGRELlE, R. R. B. Aspectos da regeneração natural de espécies arbóreas da Floresta Atlântica. Heringia, v.53, n.1, p.585-100, 2000.

FORZZA, R. C.; BAUMGRATZ, J. F. A.; BICUDO, C. E. M.; CARVAlHO JR, A. A.; COSTA, A.; COSTA, D. P. Catálogo de plantas e fungos do Brasil. Rio de Janeiro: Andrea Jakobsson Estúdio - Instituto de Pesquisa Jardim Botânico do Rio de Janeiro, 2010. 1 v. 871 p.

IBDF_INSTITUTO BRASILEIRO DE DËSENVOLVIMENTO FLORESTAL - IBDF. Padronização da nomenclatura comercial brasileira das madeiras tropicais amazônicas. Brasília, 1987. 85 p. KUHLMANN, M.; RIBEIRO, J. F.; SANTOS, D. S.; SAMPAIO, A. Época de coleta de frutos e sementes nativos para recomposição ambiental no bioma Cerrado. Documentos Embrapa Cerrados, 2018. 40 p.

LORENZI, H. Árvores brasileiras: manual de identificação e cultivo de plantas arbóreas nativas do Brasil. São Paulo: Instituto Plantarum, 2002. 368 p.

MACHADO, S. A.; SANTOS, A. A. P.; ZAMIN, N. T.; NASCIMENTO, R. G. M. Distribuição espacial de um fragmento de Floresta Ombrófila Mista Montana. Ciência Rural, Santa Maria, v. 42, n. 6, p. 1013-1019, 2012. http://dx.doi.org/10.1590/S0103-84782012005000029

MEIRA JUNIOR, M. S.; MOTA, S. L. L.; MACHADO, E. L. M.; PEREIRA, I. M. Distribuição espacial de Eremanthus incanus (Less.) Less. (Asteraceae) em duas áreas com diferentes níveis de conservação. R. bras. Bioci., Porto Alegre, v. 15, n. 1, p. 27-31, 2017.

NASCIMENTO, I. L.; ALVES, E. U.; BRUNO, R. L. A.; GONÇALVES, E. P.; COLARES, P. N. Q.; MEDEIROS, M. S. Superação da dormência em sementes de faveira (Parkia platycephala Benth.). Revista Árvore, Viçosa, v. 33, n. 1, p. 35-45, 2009.

NEGRINI, M.; AGUIAR, M. D.; VIEIRA, C. T.; SILVA, A. C.; HIGUCHI, P. Dispersão, distribuição espacial e estratificação vertical da comunidade arbórea em um fragmento florestal no Planalto Catarinense. Revista Árvore, Viçosa, v. 36, n. 5, p. 919-930, 2012. http://dx.doi.org/10.1590/S0100-67622012000500014

ODUM, E. P. Ecologia. Rio de Janeiro: Guanabara Koogan, 2010. 434 p.

ODUM, E. P. Ecologia. Rio de Janeiro: Guanabara Koogan, 1988. 434 p.

QIN, H.; DONG, G.; ZHANG, Y.; ZHANG, F.; WANG, M. Patterns of species and phylogenetic diversity of Pinus tabuliformis forests in the eastern Loess Plateau, China. Forest Ecology and Management, Amsterdam, v. 394, p. 42-51, 15 jun. $2017 . \quad$ DOI: https://dx.doi.org/10.1016/j.foreco.2017.03.030 
PEREIRA, A. A.; NETTO, S. P.; CARVALHO, L. M. T. Análise da distribuição espacial de jequitibarosa em uma Floresta Estacional Submontana. Revista Acadêmica, Curitiba, v. 4, n. 2, p. 21-34, 2006. DOI: http://dx.doi.org/10.7213/cienciaanimal.v4i2.9321

RIPLEY, B. D. Tests of randomness for spatial point patterns. Journal of the Royal Statistic Society, v. 41, n. 3, p. 368374, 1979. DOI: https://dx.doi.org/10.1111/j.25176161.1979.tb01091.x

RIPLEY, B. D. Modelling spatial patterns. Journal of the Royal Statistic Society, London, v. 39, n. 2. p. 172-212, 1977. DOI: https://dx.doi.org/10.1111/j.25176161.1977.tb01615.x

ROWLINGSON, B.; DIGGLE, P. Splancs: Spatial and spacetime point pattern analysis. $\mathrm{R}$ package version 2.01-40. Disponível em: $\quad<$ https://CRAN.Rproject.org/package $=$ splancs $>$. Acesso em: 10 Nov. 2018.

SANTANA, J. G.; NAVES, R. V. Caracterização de ambientes de cerrado com alta densidade de pequizeiros (Caryocar brasiliense Camb.) na região sudeste do estado de Goiás. Pesquisa Agropecuária Tropical, Goiânia, v. 33, n. 1, p. 1-10, 2003.

SANTOS, C. E. M.; MORGADO, M. A. D.; MATIAS, R. G. P.; WAGNER JÚNIOR, A.; BRUCKNER, C. H. Germination and emergence of passion fruit (Passiflora edulis) seeds obtained by self-and open-pollination. Acta Scientiarum. Agronomy, Maringá, v. 37, n. 4, p. 489-493, 2015. DOI: http://dx.doi.org/10.4025/actasciagron.v37i4.19616

SANTOS, H. G. dos; CARVALHO JUNIOR, W. de; DART, R. de O.; AGLIO, M. L. D.; SOUSA, J. S. de; PARES, J. G.; FONTANA, A.; MARTINS, A. L. da S.; OLIVEIRA, A. P. de. O novo mapa de solos do Brasil: Legenda atualizada. Rio de Janeiro: Embrapa Solos, 2011. 67 p. (Embrapa Solos. Documentos, 130).

SILVA, M. A. da; MELLO, M. J. de; SCOLFORO, J. R. S.; JÚNIOR, L. C.; ANDRADE, I. S.; OLIVEIRA, A. D. Análise da distribuição espacial da candeia (análise da distribuição espacial da candeia (Eremanthus erythropappus (dc.) Macleish) sujeita ao sistema de manejo porta-sementes. Cerne, Lavrasv. 14, n. 4, p. 311$316,2008$.

SONG, H.; XU, Y.; HAO, J.; ZHAO, B.; GUO, D.; SHAO, H. Investigating distribution pattern of species in a warm temperate conifer-broadleaved-mixed forest in China for sustainably utilizing forest and soils. Science of The Total Environment, Amsterdam, v. 578, p. 81-89, 2017. DOI: https://dx.doi.org/10.1016/j.scitotenv.2016.07.218

STOYAN, D.; PENTTINEN, A. Recent applications of point process methods in forestry statistics. Statistical Science, Hayward, v. 15, n. 1, p. 61-78, 2000.

TOWNSEND, C. R.; BEGON, M.; HARPER, J. L. Fundamentos em ecologia. 3.ed. Porto Alegre: Artmed, 2010. $576 \mathrm{p}$.

VIEIRA, D. S.; OLIVEIRA, M. L. R.; GAMA, J. R. V. MACHADO, E. L. M. GÖRGENS, R. B.; LAFETÁ, B. O.; GARCIA J. S. Phytosociology of a natural fragment of the floodplain forest in the Lower Tapajós River, Brazil. Bosque, Valdivia, v. 38, n. 2, p. 357-369, 2017. DOI: http://dx.doi.org/10.4067/S0717-92002017000200013 